\title{
Research on Comparisons of Micro-blog's Document Features
}

\author{
Jinyuan Zhou and Gaoyun Xia \\ Institute of Science and Technology Information of Jiangsu University, Zhenjiang, China
}

\begin{abstract}
The paper is to explore Micro-blog's features, namely, whether it belongs to Document, the paper makes comparisons on definition, elements, information processing level, similarities and differences of ten documentary information sources, features and functions between Document and Micro-blog. The paper is through literature investigation or through network research referring to Document's and Micro-blog's various features and functions. The paper concludes that Micro-blog belongs to Document. And its development and growing trend should be paid more attention. The paper is to make suggestions for Microblog's future development and management combining analysis of traditional document restrictions.
\end{abstract}

Keywords-document; micro-blog; documentary information Source; comparison

\section{INTRODUCTION}

Micro-blog is a platform for sharing, spreading and obtaining information which based on users' relationships. A user can establish personal community through Client such as WEB and WAP; and update information in 140 character blocks and realize real-time sharing. In June 2006, the birth of Twitter [1] marked the birth of Micro-blog. In 2007, the earliest Chinese micro-blog "FanFou" [2] opened to the public, which is highly similar to Twitter. Later, the four portal websites opened its Micro-blog service in sequence. With the sharp growth of Internet and computer technology, Micro-blog grows swiftly and is of great importance to our daily life, even on scientific research in future.

What is the essence of Micro-blog? Whether it can be defined as Document? What are similarities and differences between Micro-blog and Document? The paper is to answer questions above from some indexes as follows: 1) definitions; 2) elements; 3) information processing level; 4) similarities and differences of between ten documentary information sources and Micro-blog; 5) features and functions. Finally, its essence as document is proved.

\section{COMPARISONS}

\section{A. Definitions}

"Document" is a generic term of various recorded information, which can be words, symbols, pictures, audios and videos [3]. In a broad sense, Document is a carrier to record knowledge. Currently, there are numerous versions to Document's element. In accordance with the definition of General Bibliographical Description [4], "Document is a carrier to record knowledge".
Micro-blog is a kind of network information resource which includes words, pictures, audios and videos, which stored and spread through Internet. It has basic features of information resources as follows: 1 ) can be retrieved, processed and used; 2) can be counted and analyzed; 3) can be applied to the development of scientific culture and commerce.

\section{B. Elements}

There are four basic elements for Document, that is, document information, document carrier, symbol system and record mode. None of them is dispensable. Similarly, these four elements can be provided by Micro-blog.

Taking books as example, the most common document, word in books is so called document information, which can be seen in Micro-blog such as pictures and videos. The carrier of books is paper whereas Micro-blog is micro-blog app. or Internet. The symbol system of books include coding scheme such as Chinese, English, numbers, and symbols whereas Micro-blog is a kind of computer language stored in network or hardware. The record mode of books is basic printing and copying whereas Micro-blog is network blogging and recording of audio and video. Therefore, Micro-blog has the basic features of Document's four elements.

\section{Information Processing Level}

Document can be classified as Primary Source, Secondary Source and Tertiary source according to the level of information processing. Primary source includes authors' original ideas, data, principles or conclusions which derived from scientific research or social production. Those document contents are processed and saved initially by authors through certain carriers. As an information medium spread on Internet, Micro-blog is large in information numbers, various in types; also, it can record knowledge. It is novel and original; and can be processed and used by others. From descriptions above, a great majority of valuable Micro-blog information can be regarded as Primary Document.

Secondary Source is generally viewed as those sources that reprocessed, extracted and summarized on Primary source. It is a new form which derived from making Primary Sources orderly according to its external features and content characteristics. There is a part called "Topic" in Micro-blog. It means topic in "\# \#" can be seen as key words to describe present Micro-blog information content, which basically coincides with Secondary Sources' features. 
Tertiary Source is an index and/or textual consolidation of Primary and Secondary Sources. Generally, reference books such as dictionary, encyclopedia are all belong to Tertiary Source. For example, Annual Report of Chinese Micro-blog published by Shanghai Jiao Tong University annually summarizes development features of the year and looks forward to future's growing trend. From 2011, National Development Report of Politics and Law Micro-blog published by Sina Micro-blog, which makes a summary on Chinese politics and law Micro-blog. Above examples are all regeneralization and re-summarization to Secondary Source.

Furthermore, letters, notes, experimental records and conference records are all called Zero Sources, which recorded in informal physical carrier and have not been processed. A large sum of sporadic, scattered and random information on Micro-blog belongs to the Zero Source.

Micro-blog document can be original as Primary Source or Zero Source; also, it can be forwarded, discussed and added one's own opinions and analysis, which is similar to Secondary
Source. Original blog (Primary Source or Zero Source) can be rapidly positioned by users through forwarding blog. Searching and filtering to all Micro-blog information include those original and forwarded to form topics, which is similar to Tertiary Source. Users can retrieve through keywords or catalogues. It can be viewed as search engine of Micro-blog. However, the final blog still originate from Micro-blog, which is similar to Primary Source.

\section{Similarities and Differences between ten Documentary Information Sources and Micro-Blog}

Take ten Documentary Information Sources as examples, that is, books, journals, dissertations, conference papers, patents, standard literature, scientific reports, government publication, product samples, and archival document. Table 1 lists similarities and differences between valuable Micro-blog and the ten Documentary Information Sources.

TABLE I. SIMILARITIES AND DIFFERENCES BETWEEN DOCUMENTARY INFORMATION SOURCES AND MICRO-BLOG

\begin{tabular}{|c|c|c|}
\hline Comparisons & Similarities & Differences \\
\hline Books and Micro-blog & $\begin{array}{l}\text { intangible and cultural heritage of } \\
\text { humanity which has knowledge } \\
\text { value }\end{array}$ & $\begin{array}{l}\text { Books: long publication cycle } \\
\text { Micro-blog: real-time upgrades }\end{array}$ \\
\hline journals and Micro-blog & $\begin{array}{l}\text { intangible and cultural heritage of } \\
\text { humanity which has knowledge } \\
\text { value }\end{array}$ & $\begin{array}{l}\text { Journals: most of them are free } \\
\text { Micro-blog: open platform and free access }\end{array}$ \\
\hline dissertations and Micro-blog & $\begin{array}{l}\text { intangible and cultural heritage of } \\
\text { humanity which has knowledge } \\
\text { value }\end{array}$ & $\begin{array}{l}\text { Dissertations: most of them are grey literature and non-published; part of } \\
\text { published and excellent dissertations can be downloaded in database; } \\
\text { Micro-blog: open wholly }\end{array}$ \\
\hline $\begin{array}{l}\text { Conference papers and Micro- } \\
\text { blog }\end{array}$ & $\begin{array}{l}\text { intangible and cultural heritage of } \\
\text { humanity which has knowledge } \\
\text { value }\end{array}$ & $\begin{array}{l}\text { Conference papers: most of them accepted for browsing, low popularity; } \\
\text { Micro-blog: obtain information actively and passively when browsing webpage }\end{array}$ \\
\hline patents and Micro-blog & novel & Patents are more technical than Micro-blog \\
\hline $\begin{array}{c}\text { standard literature and Micro- } \\
\text { blog }\end{array}$ & Normative & Classification of standard literature are more normative than Micro-blog \\
\hline scientific reports and Micro-blog & Important information source & Scientific Micro-blogs are more personalized than scientific reports \\
\hline $\begin{array}{l}\text { government publication and } \\
\text { Micro-blog }\end{array}$ & $\begin{array}{l}\text { Blogs published by government } \\
\text { official micro-blog is similar to } \\
\text { government publication }\end{array}$ & Contents of government publication are more detailed than Micro-blogs \\
\hline product samples and Micro-blog & $\begin{array}{l}\text { Learning methods of product } \\
\text { technology, which belong to Open } \\
\text { Source }\end{array}$ & Contents of product samples are more detailed than Micro-blogs \\
\hline $\begin{array}{c}\text { archival document and Micro- } \\
\text { blog }\end{array}$ & Belong to social memory & Micro-blog pay more attention to details \\
\hline
\end{tabular}

Notices: activeness and passiveness mean a state where users obtain document

As comparisons shown in table 1 , there is a high similarity between Micro-blog and ten Documentary Information Sources. Its real-time and novel information have certain advantages when compare with books, journals and dissertations. Also, it covers information from all walks of life.

\section{E. Features and Functions}

With the continuous growth of science and technology, modern document is not traditional "books literature", that is, paper literature. At present, the modern document combines all kinds of information including computer resources and network resources. Moreover, its features change a lot simultaneously.
The main difference lies in the reform of document carrier, which changes from paper to modern electromagnetic equipment, photosensitive material and laser material. Although playing an important role in the development of Document, modern document shows some flaws in this process For instance, these flaws include accumulated in disorder, declining in quality assurance, and difficult in retrieve. Most of the time, plenty of research subjects and direction cannot be recorded timely. Therefore, the crucial decision is how to make an amendment and improvement on modern document in the process of its development and to explore a suitable way for its development. 
The birth of Micro-blog is helpful to the growth of modern document. Firstly, its information conforms to most features of modern data, for instance, tremendous in data volume, rapid in spread and increase, complicated in types. It includes various data such as daily life and scientific research. In addition, Micro-blog makes up for deficiency of modern document in some aspects as follows: 1) strong timeliness, covering current hot issues and research fronts; 2) structured contents, integrating discussion contents into together through "topic", easy to check and retrieve and improve quality wholly; 3) convenient access, without the limitation of time and space.

\section{CONCLUSION}

So far, no precise definition has been given by the official or relevant resources about whether Micro-blog belongs to Document. However, many researchers are of great interest in micro-blog research at home and abroad. In their opinion, Micro-blog and Document are in close. Besides, most functions and effects of Micro-blog are in accordance with the standards of Document. J. Chae, D. Thom, J. Yun, S. Y. Kim, T. Ertl \& D. S. Ebert think that locating information such as accurate time and space can be provided by social network service like Micro-blog[5]. In addition, it is helpful to improve their situation awareness and development for local events. Daniel Gayo Avello considers that valuable information of Micro-blog can be used as information sources [6]. Miao WANG, Youhua Liu believe that micro-blog can offer information such as focusing on competitors, searching resources, weaving social network, applying tables and making a RSS [7]. Bo YU maintains that implicit knowledge like special commercial resources can be obtained from micro-blog [8]. It will be of great importance on network direct sales, advertisement sales, mobile services and network exhibition. It is a great opportunity for many companies. Nayu YUAN insists that micro-blog is a platform and knowledge management system for information access, sharing, communication, transmission and application [9]. Using Micro-blog is a process of knowledge acquirement, management, sharing and application. Also, the process is a mutual transformation between explicit knowledge and implicit knowledge.

\section{A. The Summarization of Micro-blog}

According to comparisons of index above, micro-blog can be seen as a special document whose carrier is network. Electronic words, pictures, audios and videos can be regarded as its document information. Its code model is programming language and recording model is network blog. Also, it spread rapidly.

\section{B. Growing Trend of Micro-Blog}

Phenomena like continuously accumulated in document, slowed in search and declining in whole quality make researchers realized that modern document meets its bottleneck. As an important social network platform, micro-blog attracts document researchers for its numerous resources such as pictures, words, audios and videos. The trend of document is how to make up for the setbacks of modern document by using micro-blog information appropriately. At present, micro-blog information used only in common fields such as commercial promotion and government information publication. However, micro-blog has rarely used in scientific research. What's more, there is no official law and relevant department standardize micro-blog document, which impose restriction on its effective pigeonhole and protection.

Therefore, the trend of micro-blog document can be as follows:

- There should be a standard setting and legal norm. Standard definition should be given by relevant departments and sectors. Valuable resources should be extracted from enormous micro-blog information. Make a standard classification for its huge information. After the definition and classification, micro-blog document should be extracted and recorded by using computer technology so as to form a new kind of micro-blog electronic document.

- There should be an acceptance gained from Document fields. Authoritative database like Cnki and Wanfang whose information resources coming from journals, magazines, thesis and dissertations mostly. After standard definition and acceptance, micro-blog document can be used well by these big databases.

- Micro-blog should be applied to scientific research. Currently, micro-blog document provide service only on people's daily life for leisure and amusement mostly, and on commerce and polity rarely. The most important role document played is to promote and push the development of scientific researches and progress of humanity. The birth of micro-blog is helpful to create a new way for the development of modern document.

\section{ACKNOWLEDGMENT}

I would like to express my heartfelt thanks to my Supervisor Jinyuan Zhou whose illuminating instruction, valuable suggestions and warm encouragement has helped me greatly to shape this paper. He spent his precious time discussing and communicating with me concerning every detail of my paper. His painstaking efforts have made this paper a better one than I could ever possibly make.

I am also grateful to all the teachers who gave me lectures in the English language, and literature. Without their teaching and help, I could never have finished this paper.

I would give my thanks to the librarians of Jiangsu University who offered me warm and timely help.

\section{REFERENCES}

[1] Yanan PAN. Twitter analysis of Micro-blog[J]. Southeast Communication, 2009, 12: 117-119.(in Chinese).

[2] DCCI Internet Data Center. Chinese Micro-blog Blue Book in 2012[R]. http://news.qq.com/a/20120924/000706.htm.

[3] Ying YE, Yishan WU. Fundamental tutorial of information science[M]. Beijing Science Press, 2012. (in Chinese).

[4] General Bibliographical Description[M]. Beijing: China Standards Press, 1983. (in Chinese). 
[5] J. Chae, D. Thom, J. Yun, S. Y. Kim, T. Ertl \& D. S. Ebert. Public behavior response analysis in disaster events utilizing visual analytics of micro-blog data[J]. Computers \& Graphics, 2014(38): 51-60.

[6] D. G. Avello. Nepotistic relationships in Twitter and their impact on Rank Prestige Algorithms[J]. Information Processing \& Management, 2013(6): 1250-1258.

[7] Miao WANG, Youhua Liu. Information characteristics and its access method[J]. Modern Intelligence, 2013(01): 158-161. (in Chinese)

[8] Bo YU. Exploring information science significance of Micro-blog[J]. Library and Information Service, 2010(22): 57-60. (in Chinese)

[9] Nayu YUAN. Value analysis of Micro-blog application in library[J]. Library and Information, 2010(03): 104-106. (in Chinese) 